\title{
Correlation between Competition Trait Anxiety Structure and Paroxysmal Athletic Injuries
}

\author{
Yan Song \\ Hainan Medical College \\ 571199
}

\author{
Jiege Jiao* \\ Hainan Institute of Science and Technology \\ 571126
}

\author{
Xinyi Long \\ Hainan Medical College \\ 571199
}

\begin{abstract}
In athletic training and competition terrains, sportsmen will inevitably suffer paroxysmal athletic injuries. And anxiety is a major cause for the injuries. This article studies the correlation between competition trait anxiety structure and paroxysmal athletic injuries, assists the athletes to grasp the psychological restoration techniques, and better integrates into the normal training state. This article tentatively study the connection between competition trait anxiety in sports and paroxysmal athletic injuries; the past research data is expected to find out the internal relation between the trait anxiety and the athletic injury, provide a theoretical foundation for the sportsmen, and prevent paroxysmal sports injuries from happening. The study method in this article is qualitative research method. If the athlete has more serious stress and somatization trait anxiety, he will have greater possibility of suffering paroxysmal athletic injury. Anxiety, somatization and attention disorder can regulate the life stress and paroxysmal athletic injury.
\end{abstract}

Keywords-Anxiety; Competition Trait Anxiety; Paroxysmal Athletic Injuries

It is known that mental state can in a certain degree affect athletic performance. Studies have shown that mental factors have $20 \%$ impact on the primary and intermediate athletes, and physical reasons account for $80 \%$; while for the elite athletes, on the contrary, the mental factors can reach to $80 \%$.In recent years, it has been noted that mental state has non-negligible influence on athletic injury. At present, athletic injury is so popular that according to statistics, in sport and recreational fields, there will be 300-500 million people suffering sports injuries [1] every year. Xiang Liu, the former Olympic champion, who was failed in the Olympic Games in 2008 and 2012, had mental pressure as his key failure factor other than physical condition. Therefore, it is necessary to probe into the psychological aspects, because they have great influences on the athletic injuries; besides, it is useful to assist the athletes to grasp the mental regulations and interventions, so as to effectively control and prevent the athletic injuries.

These days, the researches on athletic injuries are mostly point to some sports related subjects such as biomechanics,

Project Source: Hainan Natural Science Foundation Project;

Project No.: 817135;

Project Name: Study on Aerobic Exercise Intervention of Cognitive Dysfunction in Aged Rats, Hippocampal Mitochondria DNA and its Related Protein Expression Mechanism physiology, and training. However, along with the deep-going study of athletic injury, it is realized that the incidence of athletic injury has influenced by the mental factors to some extent [2].Multiple impacts of athletic injuries on the sports performance can also stimulate more attentions to the cause. Some material demonstrated that $47 \%$ of the injuries were caused by mental factors which led to insufficient warming-up exercises, and $12 \%$ of the injuries were caused by action violation. It can be seen that a scientific investigation towards the mental factors can effectively prevent the athletic injuries from happening, and is favorable to help grasp the traumatized mental restoration, so that the athletes can quickly get into the training state.

\section{ANXIETY AND ATHLETIC INJURY}

\section{A. Emergence of Trait Anxiety Theory}

Anxiety is an inner discomfort which is generated when someone is subjectively has adverse consequences or potential threats, followed with some unhealthy emotions such as unease, worry and stress. In this modern society, because of more and more intense pressures, about $20 \%-30 \%$ of the ordinary people will suffer from anxiety disorders in some period of time. Mild anxiety is beneficial to us in the daily life, whereas the anxiety studying here is an intense anxiety which has deviated from its regular level. Different types of communications had been analyzed by Cattell and Scheier $(1958,1961)$ and were firstly classified into two types: state anxiety and trait anxiety. According to the statistics, from the data of elite female volleyball players in the domestic universities, the relations of these two types of anxieties showed that the correlation coefficient $\mathrm{r}=0.685$ was significantly correlated $\left(p=0.000<0.01^{* *}\right)$.And, most of the domestic and international studies believe that trait anxiety and state anxiety are correlated. After the above preliminary theory, Spielberger in 1966 put forward a more integrated theory -- state-trait anxiety. According to Spielberger's definition, trait anxiety is interpreted as relatively stable and subjective feelings in the anxiety, that is, the difference tendency to danger under a wide range of stimulus situations, which is different from state anxiety's reactivity. Spielberger then edited 'anxiety activation model' to differentiate anxieties and their functions and established 'State-Trait Anxiety Inventory (STAI)'; in this way, 
Spielberger successfully transformed 'trait anxiety' as an operable concept, terminated the philosophy history, and created a new era for quantitative analysis.

\section{B. Competition Trait Anxiety Theory}

Competition anxiety in sports is important in modern sports psychology. As an important part of psychology, competition trait anxiety can be used as a reference indicator for the athletes to adapt to the competition; besides, it can help screen the elite athletes. Jinhui Zheng once made a survey to the national aerials team players about competition anxiety the day before three international competitions in 2005 and 2006.The mean and standard deviation methods classify the competition trait anxiety into three levels, respectively advanced level (>21.61), intermediate level (15.33 to 21.61) and low level ( $<15.33)$.The calculation results show that $27 \%$ of the athletes had reached to the advanced level, 53\% the intermediate level, and $20 \%$ low level. The calculation results and the competition records show that the mild anxiety is beneficial for the athletes, assisting them to achieve good results; the reference range value to screen the elite aerials athletes is $18.47 \pm 3.14$.

Anxieties in sports competitions are often classified into three types: emotion-autonomy type, body-behavior type, and cognitive style. In the previous study, the competition trait anxiety was identified as a causal variable, and had an impact on the athletes' subjective control (Wong \& Bridges, 1995). Hui Yan analyzed the trampolinists' trait anxiety in Nanjing National Team in 2001-2002. In her study, she used the ANOVA method to analyze the above athletes' sports performances. Her analysis showed that the results $\mathrm{P}=0.006<0.01^{* *}$ were significant, which proved that the competition trait anxiety was a major causal variable; there were some other studies regarding it as an outcome variable, saying that the social support, stress training could even reduce competition trait anxiety(Smith, Smot, \&Barnettt, 1995); some others even saw it as the outcome variable of control point (Ntoumanis\&Jenes, 1998).Among multiple factors, for personality, according to the data collected from questionnaires, some studies concluded that $58.6 \%$ of the coaches and $52.2 \%$ of the guard players all believed that the personal character played an important role in the training and competitions of women's basketball team defenders. Besides, for personality, internally oriented trait anxiety is more positive, export-oriented trait anxiety is more negative; another study showed that (Kerr\&Goss, 1997), for elite athletes, there was positive correlation between trait anxiety and control points; in these four types of the people, which are high self-confirmation / high task identification, low self-confirmation / high task identification, high self-confirmation / low task identification, and low self-confirmation / low task identification, low task identification / high self-confirmation type accounts the most. Some related researches showed that competition trait anxiety is negatively correlated with the elite athletes' self-esteem (Kerr \& Goss, 1997). For example, Qufu Normal University had conducted quantitative research on 98 disabled persons in the 2005 National Disabled Bicycle Championship. The Pearson Analysis showed that there was a significant negative correlation between self-esteem and trait anxiety $\mathrm{r}=-0.513^{* *}$.

\section{Trait Anxiety and Athletic Injuries}

In the early research, Trait Anxiety Inventory (TAI) by Spielberger is mainly used in the study of trait anxiety and athletic injury, but their relation has not been explored [3].This is possibly related with that the rating scale does not point to the athletes. Therefore, when the researchers use the Competition Trait Anxiety Scale (SCAT) [4] for athletes, it was found that higher the level of the athlete, higher the incidence of athletic injuries [5-8].The investigation and mathematical statistics of 200 high competence aerobics athletes in 2004 National Aerobics and Championships showed that the degree of difficulty for high competence athletes is mainly center on 0.5-0.8, and the competition scores are directly proportional to the degree of difficulty. Therefore, if the athlete wants to get good results in competition, he will increase the degree of difficulty, which will upped the risk of athletic injury.

In the 1990s, Smith and the other two researchers compiled a table to measure the sport trait anxiety -- 'sport trait anxiety scale' (SAS).This table effectively differentiates trait anxiety into three types: Somatization sport trait anxiety (somatization), Anxiety sport trait anxiety (anxiety), Attention to disorder sport trait anxiety (attention disorder).For somatization and anxiety, Lichun Li and etc. once used this table to measure 243 athletes and their sports injuries within a year; the analysis showed that OR values of anxiety and somatization were respectively $1.782\left(\mathrm{P}<0.05^{*}\right)$ and $1.381\left(\mathrm{P}<0.05^{*}\right)$.The results showed that when other factors are stable, the higher the athlete's anxiety and somatization, the greater the risk of paroxysmal athletic injuries. For attention disorder, Stress-Injury Theory believes that attention disorder is also a fundamental cause of athletic injury. Therefore, the study of attention disorder is significant to athletic injury. Besides, Jones and Swain also put forward a view of Anxiety Directionality, evaluating whether the athlete's self-assessment of anxiety to the sport is positive or negative. A questionnaire survey on 88 athletes in East China Normal University and the statistics of their competition results showed that for cognitive anxiety $(\mathrm{F}(1,86)=3.92, \mathrm{P}=0.0482)$, the anxiety reaction of high competence athletes is more positive than that of low level athletes.

1) Sharpened Trait Anxiety Easy Lead to Paroxysmal Athletic Injury

Sharpened trait anxiety hinders the normal emotion, and is accompanied by physical discomfort, which hinders normal psychological activity. The athlete will have some related symptoms such as muscular tension, aprosexia, and smaller vision. Youru Yu and etc. once made an investigation towards 1091 athletes who had been injured in recent three years in Jiangxi Province, and the study showed that due to psychological anxiety, 439 athletes were injured, which accounted for about $40.2 \%$ of the total. Mascular tension also leads to some psychological changes, such as ataxia, decreased dexterity of action and accuracy, and poor adaptability to the surrounding environment, and it is to a large extent, can lead to athletic injury.

The athlete, if he is on the strain, and especially with negative self-assessment, will often make unconscious 
protective behaviors in training competitions, which is also a potential factor to the incidence of the athletic injury. The athletes will usually has potentially dangerous threat in learning new technology, and this threat will produce anxiety and other negative spirits to those low level athletes in practice match because of the passive incompletion of action, psychological and physical energy consumption; and, if the athlete has spent too much psychological and physiological energy, he will easily suffer athletic injury[9].A survey of 121 track and field athletes in Henan province has found that 78 injuries are caused by technical errors because of tension, which accounts for $64.5 \%$ of the total injuries; 85 of the injuries are caused by unskillful movements in high-degree difficulty, which accounts for $70.3 \%$ of the total.

2) Relation between Multi-dimensional Trait Anxiety and Paroxysmal Athletic Injury

Presently, there is a relatively positive relationship between competition trait anxiety and athletic injury. Contemporary researchers believe that competition trait anxiety has a multi-dimensional structure. In the field of sports psychology, we also believe that competition trait anxiety has a multi-dimensional structure [10].A number of studies used 'sports anxiety scale' to measure the athletes' multi-dimensional competition trait anxiety. The questionnaire survey of high competence swimming athletes in 90 colleges and universities in Shandong province showed that the anxiety's variance value, gender $(\mathrm{F}=3.329, \mathrm{P}=0.040)$, age $(\mathrm{F}=5.450, \mathrm{P}=0.006)$, training time $(\mathrm{F}=3.849, \mathrm{P}=0.024)$, athletic performance $(\mathrm{F}=6.930$, $\mathrm{P}=0.003$ ) were significantly correlated with trait anxiety. There are also some researches on the relationship between different sports levels and trait anxiety, for example, Hunan Normal University had conduct an investigation of 61 Trampoline Game athletes from 11 provinces and cities in Ninth Games; the results showed that the sports levels had a significant influence on the athletes' confidence $\left(\mathrm{p}=0.013<0.05^{*}\right)$, among which the higher the level of trait anxiety, the lower performance of the trait anxiety. For those people with different training periods, the longer the training period, the less the concern of anxiety sport trait anxiety and somatization sport trait anxiety; for the relationship study about age groups and anxiety, for example, Suzhou University had conducted an investigation of 23 women volleyball team players in 2005 National College of Xinghua Cup Volleyball Match, it was shown that the age was not directly proportional to trait anxiety; and, the diversity in $<23$ year-old volleyball players was higher than that of $\geq 23$ year-old ones; however, this diversity was not significant $\left(\mathrm{P}=0.118>0.05^{*}\right)$; the standard deviation analysis showed that the trait anxiety values in athletes in $<23$ year old was 9.28, the discrete degree was higher than that of $\geq 23$ year old players, whose trait anxiety was 7.81 . It also shows that the anxiety levels have fluctuated more obviously in most of the younger athletes.

As somatization reflects the physiological response patterns, it directly affects the incidence of athletic injuries; as anxiety reflects the athletes' cognitive assessment model, it can not only regulate the stress in the athletic injury process, but also directly influent the incidence of athletic injury.

\section{CONCLUSIONS AND SUGGESTIONS}

\section{A. Conclusions}

If the athlete has more serious stress and somatization trait anxiety, he will have greater possibility of suffering paroxysmal athletic injury. The athlete's anxiety sport trait anxiety can better adjust his negative life and take effect on his paroxysmal athletic injury.

Anxiety, somatization and attention disorder can regulate the life stress and paroxysmal athletic injury. Considering the influencing factors of athletic injury, it is concluded that the anxiety sport trait anxiety is relatively higher, which is not only related to the athlete's susceptibility to acute athletic injury, but also regulate the variables. The anxiety sport trait anxiety is an emotional tendency which is caused by individual's negative assessment to his self-ability or the negative expectation of the competition result. Therefore, this anxiety affects the athletes' stress response to the sports surrounding, and hereafter has a direct impact on the athletic injury.

\section{B. Suggestions}

It is necessary to understand athletes' competition trait anxiety in advance, adopt the corresponding psychological trainings, take various measures to alleviate competition trait anxiety, and diminish the risk of athletic injury.

It is needed to strengthen the athletes' cultural education, adjust their cognitive structures, improve their psychological anxiety state, and avoid acute athletic injuries.

It is urgent to provide the appropriate psychological trainings for the athletes, so that they can treat the game and some possible situations fairly and scientifically; in this way, the paroxysmal athletic injuries caused by relatively more intense competition trait anxiety in sports can be prevented.

\section{REFERENCES}

[1] Xue Yi, Guoyi Ma. Psychological analysis in athletic injuries [J]. Journal of Hebei Institute of Physical Education, 2004 (3).

[2] Liu Ji. Sports psychology [M]. Higher education press, 2006122-134.

[3] [3] Spielberger CD,Gorsuch R,Lushene R. Manual for the state trait anxiety inventory [M]. Polo Alto. CA: Consulting Psychologists Press.1970.

[4] Martens R.Sport competition anxiety test [Z].Champaign. IL: Hu-man Kinetics, 1977.

[5] Petrie TA Coping skills. competitive trait anxiety and playing 5Iulus moderating effects on the life stress-injury relationship[J]Journal of Sport and Exercise Psychology,1993,15:261-274.

[6] Hauson SJ, McCullag P, Tonymon P. The relationship of personality characteristics life stress and coping resources to athletic injury [J].Journal of Sport and Exercise Psychology. 1992. 1 4:262-272.

[7] Lavllee L. The relationship of stress, competitive anxiety, mood state, and social support to athletic injury[J]. Journal of Athletic Training, 1996, 31(4)296-299.

[8] Blackwdl B, McCullagh P. The relationship of athletic injury to life stress. Competitive anxiety and coping resources [J].Athletic Training, 1990 25:23-27.

[9] Ford IW,Eklund RC.Gordon DJ The examination of psychosocial variables moderating the relationship between life stress and injury time-loss among athletes of a high standard [J] Journal of Sports Science, 2000, 18:301-312. 
